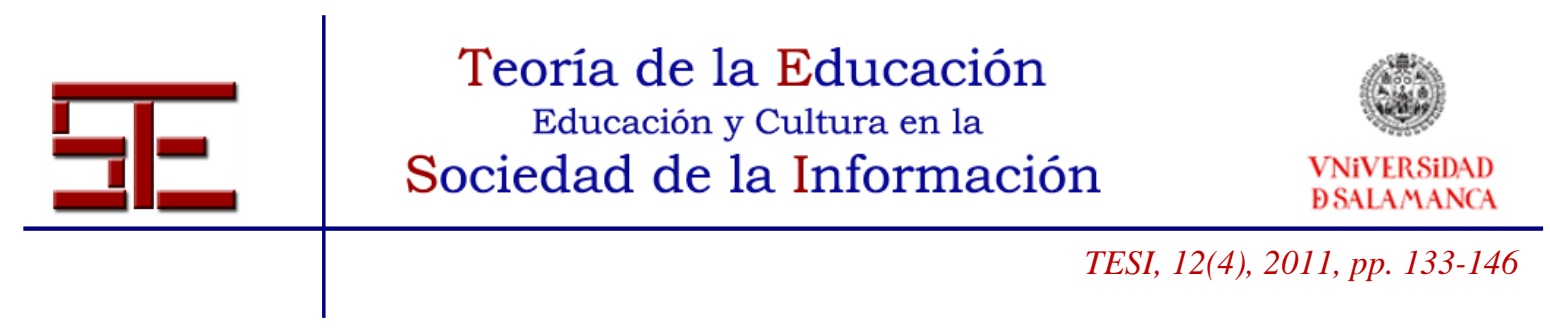

\title{
EXPERIENCIAS DOCENTES DE TRABAJO COLABORATIVO EN DISTINTAS ÁREAS DE CIENCIAS
}

Resumen: Se comparan los resultados de las experiencias docentes colaborativas desarrolladas en asignaturas de diferentes ramas de ciencia. El planteamiento inicial es común: involucrar de forma activa al estudiante en su proceso de aprendizaje. La metodología del trabajo en colaboración fue particularizada según los objetivos de cada asignatura y el nivel de los estudiantes. Como resultado del trabajo se destaca la respuesta favorable y la mayor participación de los estudiantes mediante la incorporación de actividades de estudio colaborativo.

Palabras clave: Autoaprendizaje; enseñanza en equipo; trabajo colaborativo en grupo; herramientas tecnológicas. 


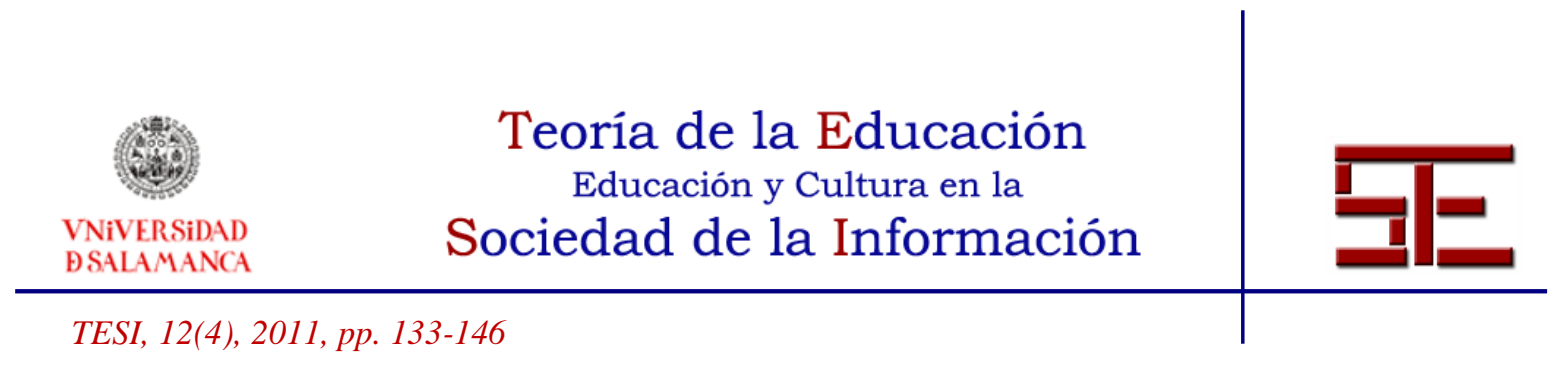

\title{
COLLABORATIVE TEACHING EXPERIENCES OF SCIENCE IN DIFFERENT AREAS
}

\begin{abstract}
We compare the experiences about collaborative activities carried out in different matters of science degrees. The common starting point is focused on getting students actively involved in the learning process. However, different methods were applied according to some specifics objectives and student levels. As result of this work we bring out the students favorably response and higher engagement by using collaborative activities.
\end{abstract}

Keywords: Self instruction; team teaching; collaborative work; technological toolbox.

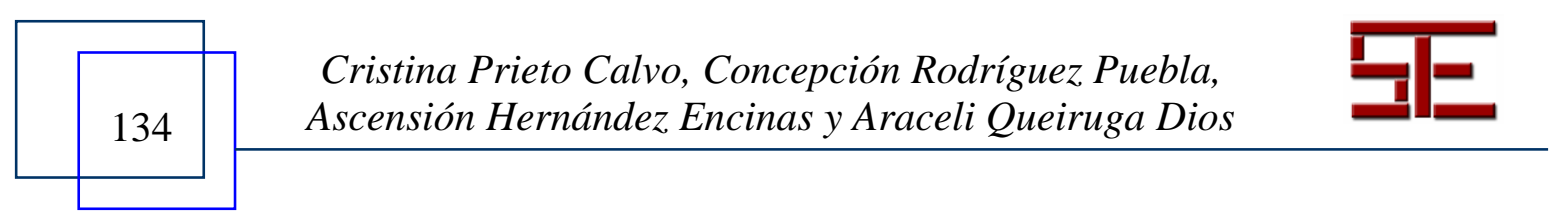




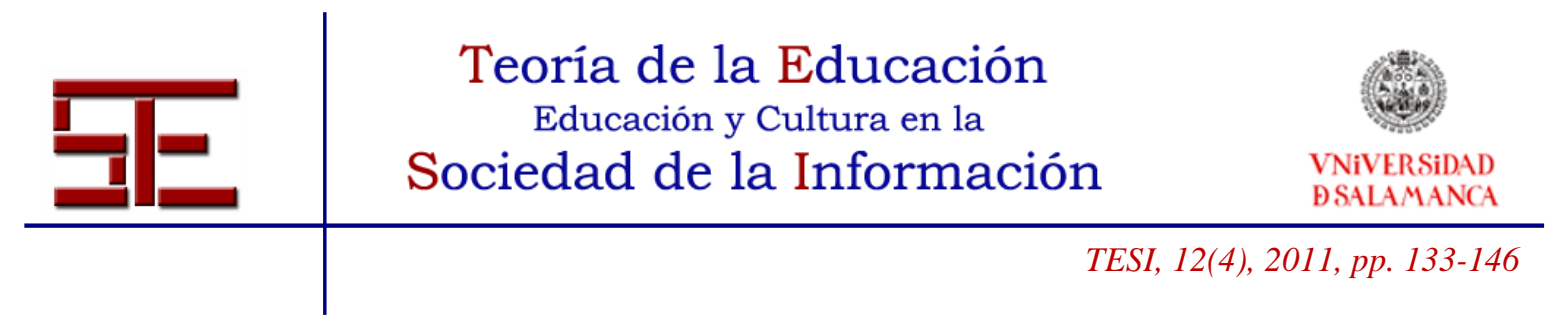

\section{EXPERIENCIAS DOCENTES DE TRABAJO COLABORATIVO EN DISTINTAS ÁREAS DE CIENCIAS}

Fecha de recepción: 31/10/2011; fecha de aceptación: 18/11/2011; fecha de publicación: 20/12/2011

Cristina Prieto Calvo

cprieto@usal.es

Universidad de Salamanca

Concepción Rodríguez Puebla

concha@usal.es

Universidad de Salamanca

Ascensión Hernández Encina

ascen@usal.es

Universidad de Salamanca

Araceli Queiruga Dios

queirugadios@usal.es

Universidad de Salamanca

\section{1.- INTRODUCCIÓN}

Uno de los retos básicos de la educación actual es preparar a las personas para ser capaces de participar plenamente en una sociedad de la información en que el conocimiento es fuente crítica de desarrollo social y económico (Guitert et al., 2007)

El trabajo en equipo cobra gran importancia en el EEES. En primer lugar, como metodología que facilita la adquisición de competencias mediante procesos de trabajo activo y participativo. En segundo lugar, porque el trabajo en equipo es en la actualidad una de las habilidades más valoradas en los entornos profesionales.

Aunque en la concepción y diseño de los nuevos grados se ha tenido muy en cuenta el logro de competencias directamente relacionadas con el futuro profesional del estudiante, a menudo los alumnos consideran cada materia como independiente de la formación previa y tienen grandes dificultades en reconocer su aplicación en el mundo actual.

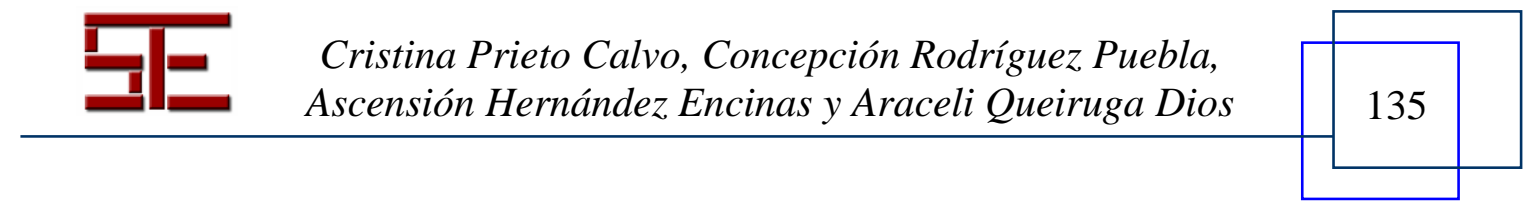




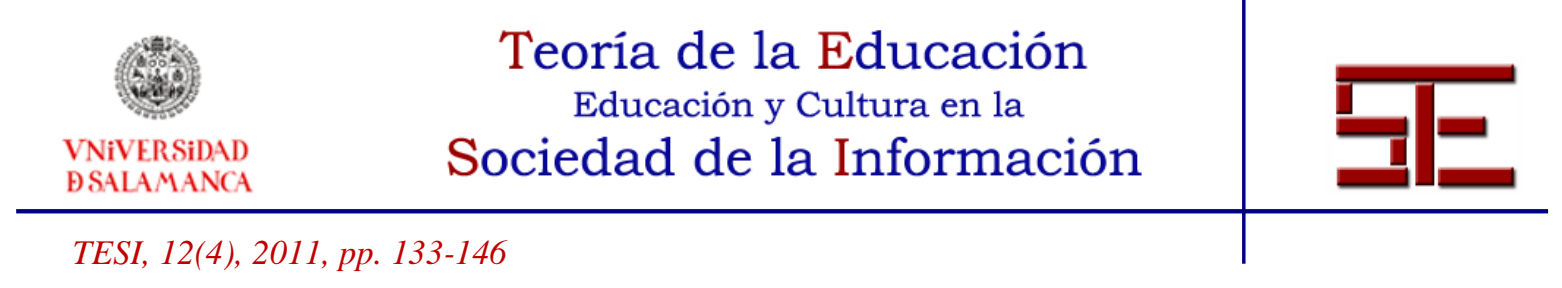

Con objeto de que el estudiante verifique efectivamente la aplicación de la materia explicada en lo que será su ámbito de trabajo se ha diseñado, en tres asignaturas diferentes de otras tantas áreas de ciencias, actividades colaborativas en las que, como en la sociedad actual, la información y la comunicación desempeñan un importante papel.

Mediante el trabajo en grupo no sólo se trata de potenciar las habilidades de gestión de la información (búsqueda, selección, análisis, tratamiento, interpretación y estructuración), sino también las competencias transversales de organización en un trabajo colaborativo. En éste se utiliza la formación de grupos para que el estudiante comprenda y aprenda mejor una materia, interaccionando con sus compañeros de equipo. Las actividades que combinan TIC y nuevas metodologías docentes facilitan el aprendizaje y permiten el desarrollo de capacidades de organización, toma de decisiones, trabajo en grupo y aplicación de conocimientos en la práctica. Además, y muy importante, suponen una considerable mejora en el grado de motivación del alumno.

El trabajo colaborativo permite también el ejercicio del espíritu crítico, que se desarrolla y proyecta hacia la crítica constructiva. Esta misma actitud crítica es la que se ejerce en el proceso de evaluación de las competencias conseguidas en cualquier tarea docente programada. Siguiendo a Martínez y Carrasco (2006) la evaluación es un medio para un fin, siendo su propósito más importante guiar y ayudar a aprender. Por ello, el entrenamiento de los alumnos en las tomas de decisiones en el grupo de trabajo les prepara para la evaluación de sus propios compañeros y por comparación les estimula para la realización de un trabajo bien hecho.

\section{2.- OBJETIVOS}

La actividad propuesta pretende que los estudiantes adquieran nuevos conocimientos y competencias que de otra forma no llegarían a adquirir. Para ello se realizan trabajos en grupo, utilizando programas adecuados, lo que facilita el aprendizaje cooperativo y colaborativo.

Los objetivos se pueden agrupar según sus características en:

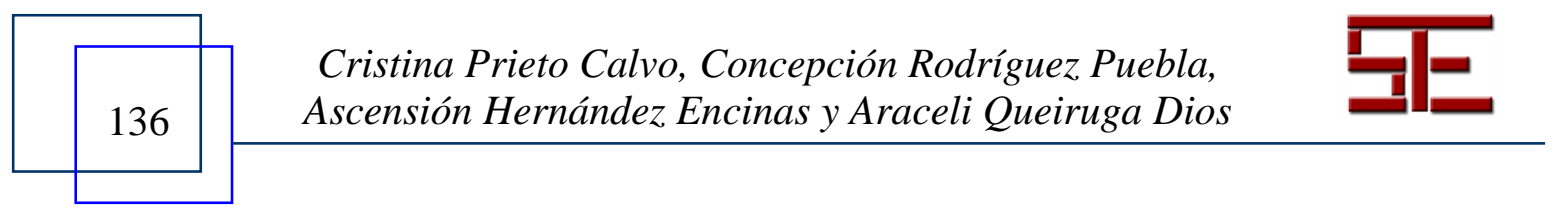




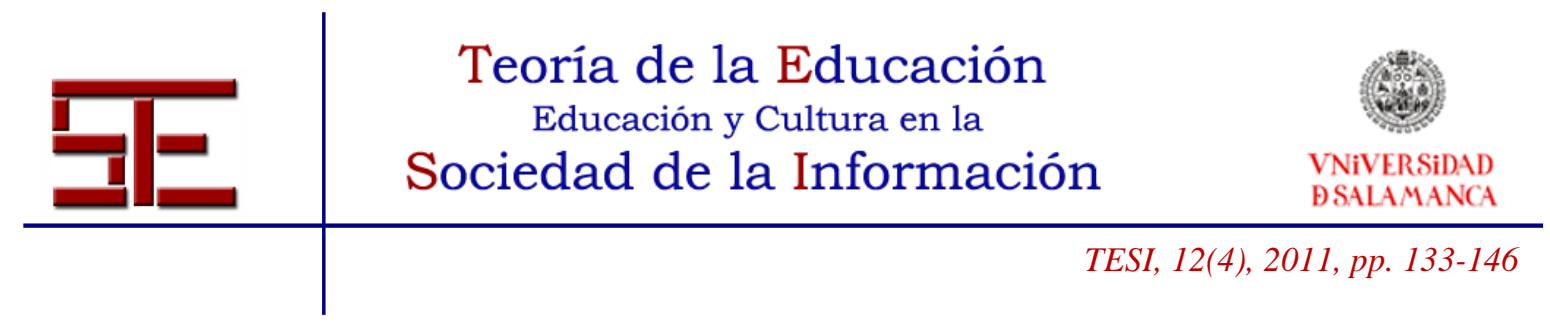

Conceptuales:

- Obtener la capacidad de síntesis necesaria para plasmar su trabajo en un póster tipo congreso científico y/o una presentación informática, sabiendo estructurar el trabajo para conseguir comunicar todo lo necesario, con rigor y concisión.

- Motivar el aprendizaje de las asignaturas desde un punto de vista de la aplicación de los conocimientos.

Actitudinales:

- Adquirir destrezas en la búsqueda de información relevante, utilizando las bases de datos bibliográficas, lo que puede servir como introducción a posibles trabajos de investigación. Utilizar las TIC para el buen desarrollo del trabajo. En general el uso de las TIC es un elemento motivador para el estudiante.

- Potenciar los debates entre los compañeros de equipo para llegar a una meta común, respetando las ideas de los demás y promoviendo la sana rivalidad entre ellos. Promover el espíritu crítico constructivo, disciplina de grupo, la discusión y el diálogo.

- Desarrollar las aptitudes de comunicación oral tanto a público especialista como no especialista.

Para conseguir estos objetivos los docentes llevan a cabo las siguientes actividades:

- Preparar recursos docentes teóricos y experimentales, definir los temas y en algunos casos asignar el reparto de las tareas.

- Dirigir la búsqueda de información y plantear cuestiones o motivos de interés para desarrollar el trabajo. Además de fomentar discusiones durante las exposiciones para profundizar en el conocimiento de los temas.

\section{3.- METODOLOGÍA}

El cambio de paradigma educativo que implica el EEES supone un proceso de enseñanza-aprendizaje en que el estudiante cobra protagonismo, ganando de forma progresiva autonomía en su formación bajo la guía del profesor y la cooperación con sus compañeros. El trabajo colaborativo incorpora actividades que el profesor/a realiza con sus estudiantes en clase y actividades que los estudiantes realizan en grupos fuera de clase. En el Cuadro 1 se resumen las metodologías docentes empleadas en algunas de nuestras asignaturas. Se han ordenado de forma que el papel activo del estudiante en cada una de ellas va creciendo de izquierda a derecha.

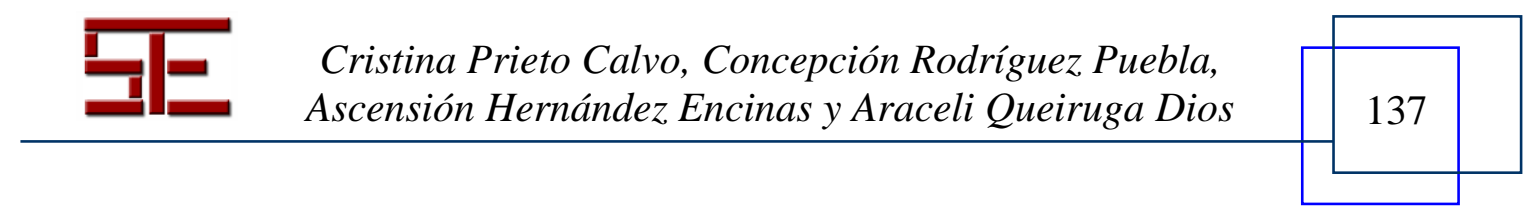




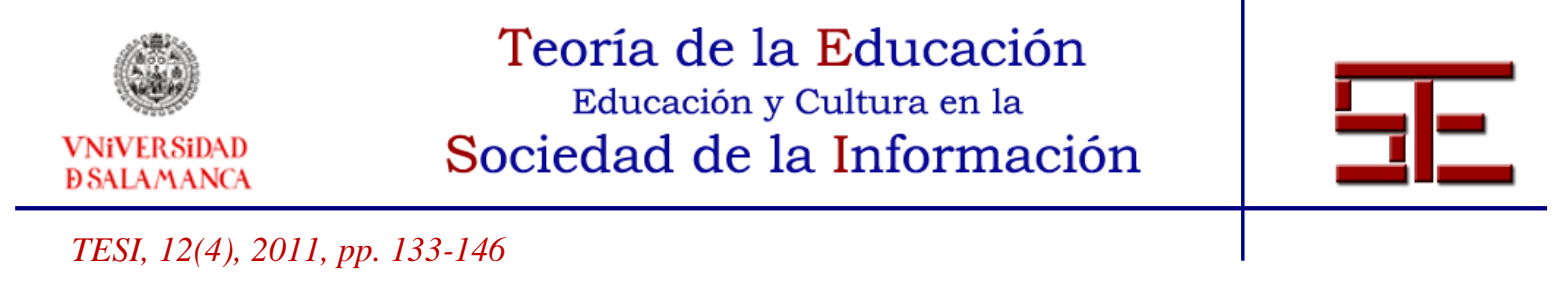

\begin{tabular}{|c|c|c|c|c|c|}
\hline \multicolumn{6}{|c|}{ Enseñanza/aprendizaje mediante trabajos de colaboración } \\
\hline \multirow{2}{*}{ Método } & \multicolumn{4}{|c|}{ Colaboración entre profesor y estudiante } & Estudiantes \\
\hline & Teoría & Práctica & Tareas & Propuesta trabajos & Realización \\
\hline Evaluación & \multicolumn{2}{|c|}{$\begin{array}{c}\text { Exámenes finales } \\
40 \%-60 \%\end{array}$} & $\begin{array}{c}\text { Calificación } \\
10 \%-20 \%\end{array}$ & $\begin{array}{c}\text { Presentaciones } \\
20 \%-30 \%\end{array}$ & $\begin{array}{c}\text { Participación } \\
0 \%-10 \%\end{array}$ \\
\hline
\end{tabular}

Cuadro 3: Diseño de las actividades del trabajo colaborativo

Además de las clases teóricas y prácticas, se asignan tareas para que el estudiante trabaje también de forma individualizada.

La colaboración entre profesor y estudiante se desarrolla tanto a través de la plataforma Studium (https://moodle.usal.es/) como de forma personal, para notificar la calificación y las oportunas correcciones. Una vez que los estudiantes se familiarizan con la metodología de aprendizaje, se proponen los temas de trabajo colaborativo que se seleccionan adecuadamente para que complementen diversas partes de la asignatura y hagan llegar al estudiante la importancia de la metodología de investigación.

Las actividades que forman el conjunto de la experiencia se han desarrollado en las asignaturas de Física (Licenciatura en Biotecnología), Climatología (Licenciatura en Física), Geología y Climatología (Grado de Ingeniería Agroalimentaria) y Complementos de Matemáticas (Ingeniería Industrial). Las tres materias difieren tanto en el nivel dentro del plan de estudios correspondiente como en el número de alumnos y el carácter de la asignatura. En el Cuadro 2 se presenta un resumen de las características más importantes que pueden afectar al desarrollo de la experiencia.

La columna "Actividades desarrolladas" muestra los distintos tipos de manifestación que se incluye en la actividad mediada por las TIC y la columna \% se refiere al porcentaje del trabajo en grupo sobre la nota final.

\begin{tabular}{|c|c|c|c|c|c|c|c|c|}
\hline \multirow{2}{*}{ Materia } & \multirow{2}{*}{ Titulación } & \multirow{2}{*}{$\begin{array}{l}\text { Carácter } \\
\text { (créditos) }\end{array}$} & \multirow{2}{*}{ Curso } & \multirow{2}{*}{$\begin{array}{c}N^{o} \\
\text { alum } \\
\text { nos }\end{array}$} & \multicolumn{3}{|c|}{$\begin{array}{c}\text { Actividades } \\
\text { desarrolladas }\end{array}$} & \multirow[t]{2}{*}{$\%$} \\
\hline & & & & & Póster & $\begin{array}{l}\text { Presen } \\
\text { tación }\end{array}$ & $\begin{array}{c}\text { Expos. } \\
\text { oral }\end{array}$ & \\
\hline Física & Biotecnología & $\begin{array}{l}\text { Troncal } \\
\text { (6) }\end{array}$ & $1^{\mathrm{o}}$ & 30 & Sí & Sí & Sí & 20 \\
\hline
\end{tabular}

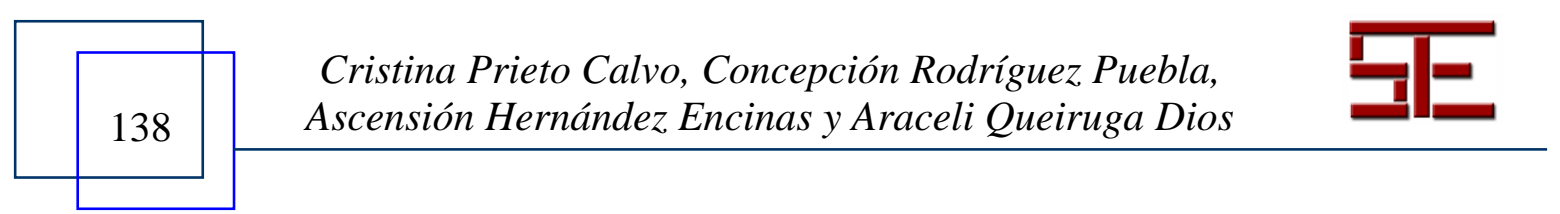




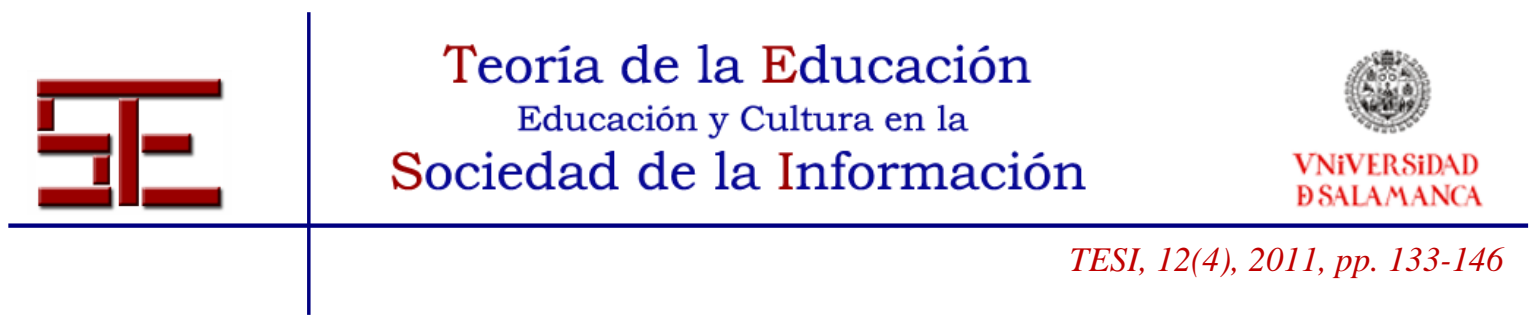

\begin{tabular}{|c|c|c|c|c|c|c|c|c|}
\hline Climatología & Físicas & $\begin{array}{c}\text { Optativa } \\
(6)\end{array}$ & $4^{\circ}$ & 30 & Sí & Sí & Si & 30 \\
\hline $\begin{array}{c}\text { Geología y } \\
\text { Climatología }\end{array}$ & $\begin{array}{c}\text { Ingeniería } \\
\text { Agroalimentaria }\end{array}$ & $\begin{array}{c}\text { Básica } \\
(9)\end{array}$ & $1^{\circ}$ & 15 & No & Si & Si & 30 \\
\hline $\begin{array}{c}\text { Comp. de } \\
\text { Matemáticas }\end{array}$ & $\begin{array}{c}\text { Ingeniería } \\
\text { Industrial }\end{array}$ & $\begin{array}{c}\text { Optativa } \\
(6)\end{array}$ & $4^{\circ}$ & 8 & Sí & Sí & Sí & 30 \\
\hline
\end{tabular}

\section{1.- Diferencias y semejanzas entre las distintas áreas de conocimiento}

Algunos de los aspectos comunes de la experiencia realizada en las áreas de ciencias en las que se desarrolla esta labor docente son los siguientes:

- Sobre el temario de la asignatura se propone a los estudiantes la realización de trabajos en grupo (evaluables) y se les comunican las condiciones en que se llevarán a cabo. Se les sugiere la necesidad de seguir un calendario de trabajo para que los resultados sean fructíferos y no se conviertan en un cúmulo de información reunida a última hora.

- En algunos casos los estudiantes eligen el tema sobre el que trabajarán. En todos los casos ese tema debe estar relacionado con la aplicación de la materia en la sociedad actual. Normalmente, al afrontar el trabajo, los alumnos tienen conocimientos suficientes de la asignatura y seleccionan el tema por afinidad y preferencias personales.

- Los estudiantes se agrupan libremente, en grupos pequeños de 2 a 5 miembros, y en general el profesor no interviene en la dinámica del grupo, siendo los mismos alumnos los que establecen sus pautas de organización y reparto de tareas.

- Se proporciona a los estudiantes un documento de trabajo con las orientaciones y ayuda para llevar a cabo las diferentes partes del proyecto, pero la realización del trabajo es libre, siempre con las directrices y supervisión del profesor, que realiza tareas de tutorización tanto a través de las TIC como de forma presencial.

- Como resultado material de sus actividades, cada grupo debe realizar un póster científico y/o presentación informática que servirá de apoyo en la comunicación oral de sus investigaciones al resto de compañeros. Un aspecto muy importante de la exposición pública es la capacidad de respuesta ante las preguntas que formulan los oyentes. Asimismo, cuando se invierten los papeles, la capacidad para generar preguntas da idea del grado de madurez que se ha logrado en la materia.

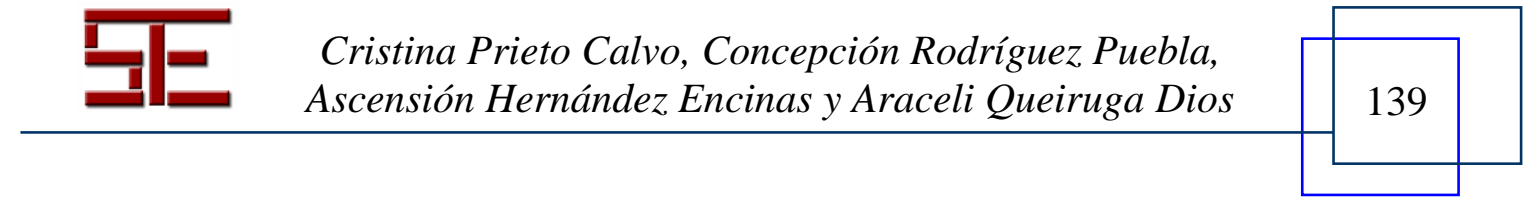




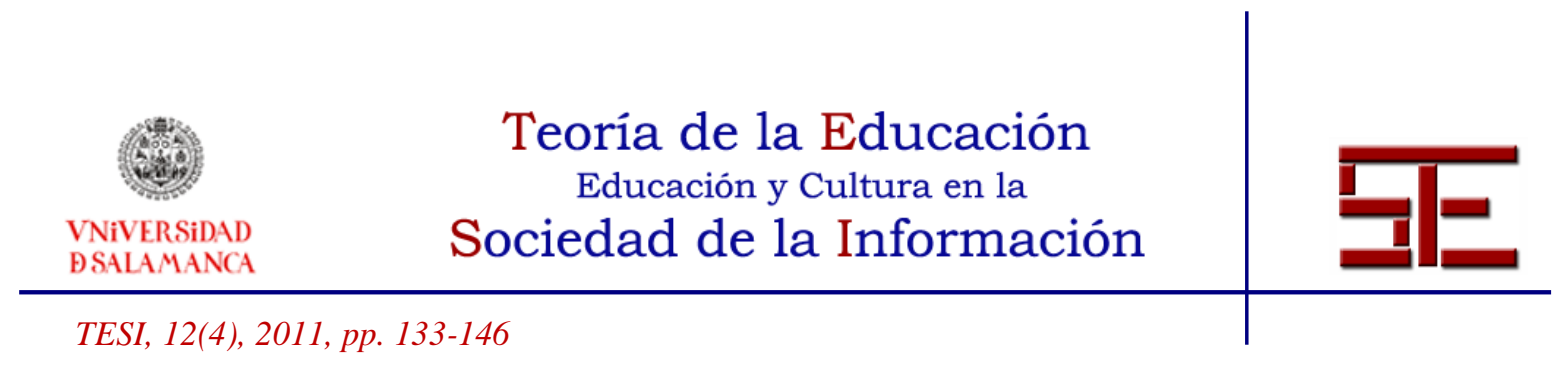

- En algunos casos, los estudiantes han participado en la evaluación de sus compañeros, una vez que se han completado las correspondientes exposiciones. También se han elaborado encuestas para que los alumnos expresen por escrito sus opiniones sobre distintos aspectos de los trabajos realizados.

- Se utiliza la plataforma Studium para la entrega de documentación, asignación de tareas, planteamiento de cuestionarios y propuesta de foros de discusión para comunicar noticias e incluir bibliografía y novedades que orienten los trabajos de colaboración.

Algunas diferencias que observamos son las siguientes:

- Las herramientas tecnológicas y programas que utilizan los estudiantes varían dependiendo del área de estudio (PowerPoint, Open Canvas, PhotoShop, Mathematica, GrADS). La utilización de cualquiera de estos programas permite a los estudiantes colaborar para obtener el resultado final esperado (Queiruga et al., 2011).

- Los niveles académicos implicados en este estudio son diferentes: se imparte docencia a alumnos de $1^{\circ}$ curso de grado en Biotecnología, $1^{\circ}$ de Ingeniería Agroalimentaria, $4^{\circ}$ de licenciatura en Ciencias Físicas y $4^{\circ}$ de Ingeniería Industrial. En principio, se espera que haya diferencias no sólo para las distintas titulaciones, sino también por los diversos niveles, grado de madurez, motivación por aprender y necesidad de una buena nota.

\section{4.- RESULTADOS OBTENIDOS}

Los estudiantes han mostrado destreza en la búsqueda de información bibliográfica o en Internet. El profesor les ha ayudado fundamentalmente a limitar sus ambiciones en el tema en cuestión: un póster o un trabajo como el propuesto no pretende ser un tratado científico. Ningún grupo tuvo dificultades en el uso de las TIC. Sin embargo, sí les resultó más difícil tener que limitarse a lo que se puede adaptar, claramente, al tamaño del póster. La orientación sobre los aspectos más importantes del tema y su organización supuso la parte más laboriosa en el trabajo del profesor.

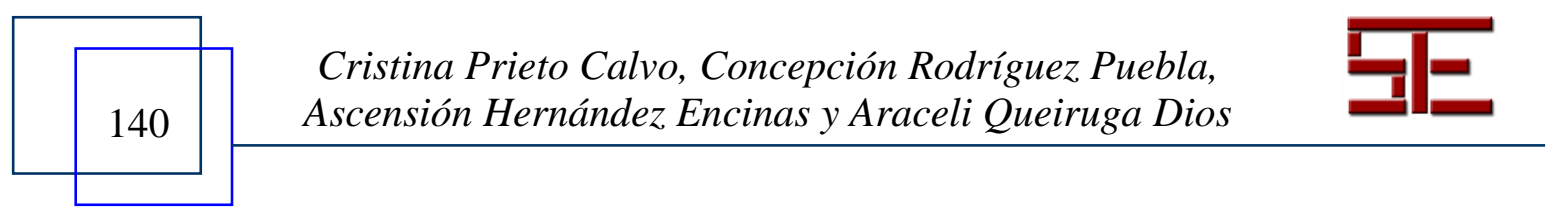




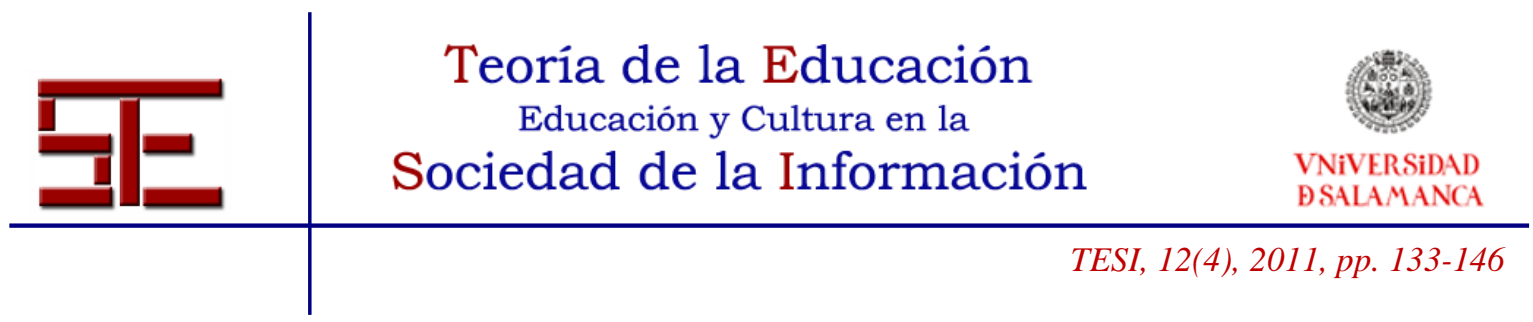

\section{1.- Biotecnología}

Como punto de partida los estudiantes realizan una foto en que se muestra un fenómeno físico. El objetivo final es que los estudiantes, relacionando dicho fenómeno con su ámbito de trabajo, constaten y sean capaces de transmitir los vínculos que en la actualidad existen entre disciplinas a priori desconectadas, como la física básica y las ciencias de la vida (biología, biotecnología, medicina...).

La fotografía es realizada por los propios estudiantes, bien de una situación que aparece espontáneamente o de una situación que ellos han preparado con el objetivo de resaltar el fenómeno que les interesa. Se les recomienda que, ya que la foto será el reclamo de un cartel, sea visualmente atractiva.

En el segundo paso de la actividad los estudiantes han elaborado un póster tipo congreso científico analizando la física reflejada en la fotografía e ilustrando su manifestación en el campo de la Biología. A pesar de la diversidad en la formación inicial de los estudiantes, espontáneamente se distribuyeron de forma que ningún grupo veía limitada su elección de tema por falta de conocimientos anteriores.

La experiencia se ha desarrollado en el primer cuatrimestre del curso, y la distribución temporal de las actividades ha sido la siguiente:

- Primera semana de noviembre: formación de los grupos (los estudiantes se agrupan libremente), elección del tema para su fotografía, posibilidades para la aplicación biotecnológica...

- Tercera semana de noviembre: tutoría con cada grupo para discutir el planteamiento de su trabajo y la organización del póster.

- Primera semana de diciembre: versión definitiva de los pósteres para mandar a imprimir.

- Segunda semana de diciembre: exhibición de los carteles y presentación pública de los trabajos por los miembros de cada grupo.

Tanto en la elaboración de las presentaciones como de los carteles se ha utilizado MS Powerpoint, ya que resulta familiar y accesible a la mayoría de los estudiantes. La impresión de los carteles, en tamaño $50 \times 70 \mathrm{~cm}$, se ha hecho mediante subvención del proyecto de la Universidad de Salamanca ID/073.

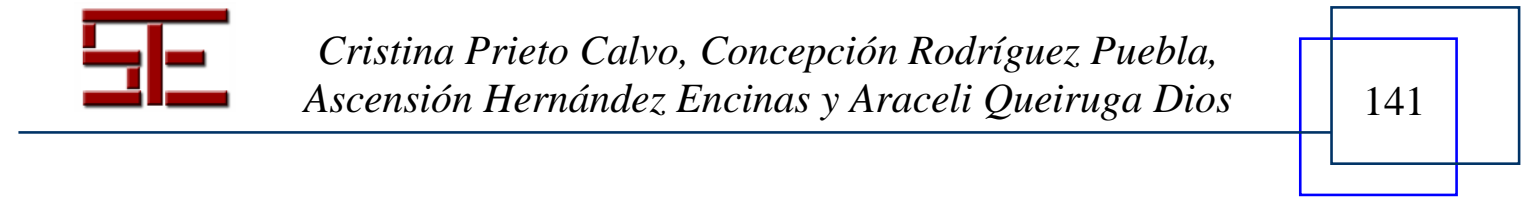




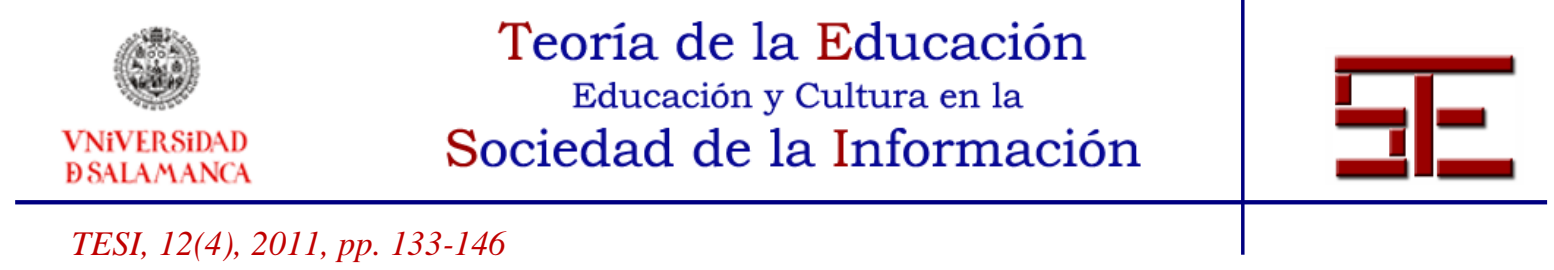

En general la preparación de la fotografía fue cuidadosa, buscando siempre un resultado vistoso por sus colores y composición estética. Curiosamente, aunque los intereses de los estudiantes de Biotecnología se dirigen hacia los aspectos relacionados con las ciencias de la vida, sus mayores dificultades radicaron en encontrar aplicaciones biotecnológicas de las que pudieran desentrañar la física subyacente. Una muestra del trabajo realizado por los alumnos puede observarse en la Figura1, al final de esta sección.

Los estudiantes han mostrado destreza en la búsqueda de información bibliográfica o en Internet. El profesor les ha ayudado fundamentalmente a limitar sus ambiciones en el tema en cuestión: un póster o un trabajo como el propuesto no pretende ser un tratado científico. Ningún grupo tuvo dificultades en el uso de MS Powerpoint para la elaboración del póster o de la presentación. Sin embargo sí les resultó más difícil tener que limitarse a lo que se puede acomodar claramente en un cartel de $50 \times 70 \mathrm{~cm}$ que contiene una foto de $20 \times 30 \mathrm{~cm}$ como protagonista. La orientación sobre los aspectos más importantes del tema y su organización supuso la parte más laboriosa en el trabajo del profesor.

Para completar la actividad con el desarrollo de competencias de comunicación oral se hicieron mesas redondas en que cada grupo, mediante una presentación informática, exponía públicamente su trabajo. El tiempo asignado para cada presentación fue de 15 minutos. Todos los miembros del grupo debían participar en la misma. Los compañeros de otros grupos estaban obligados a hacer preguntas sobre el tema. La participación de los alumnos en los turnos de preguntas fue entusiasta, estableciendo debates animados sobre la relación de los distintos temas con aspectos científicos de actualidad y demostrando la atención prestada a sus compañeros. Por su parte, los ponentes en cada caso demostraron su dominio del tema correspondiente, más allá de lo mostrado en el póster o presentación.

Como parte del proceso formativo, se pidió a los estudiantes que participaran en la calificación del trabajo de los demás grupos. Para ello se les proporcionó una encuesta anónima en que cada estudiante individual calificaba al resto de los grupos. Se puntuaban aspectos como la apariencia general del póster, el interés del tema, la idoneidad de la aplicación biotecnológica, la eficacia en la comunicación oral o el acierto en las respuestas a las preguntas formuladas. En la misma encuesta se incluyó una zona para observaciones.

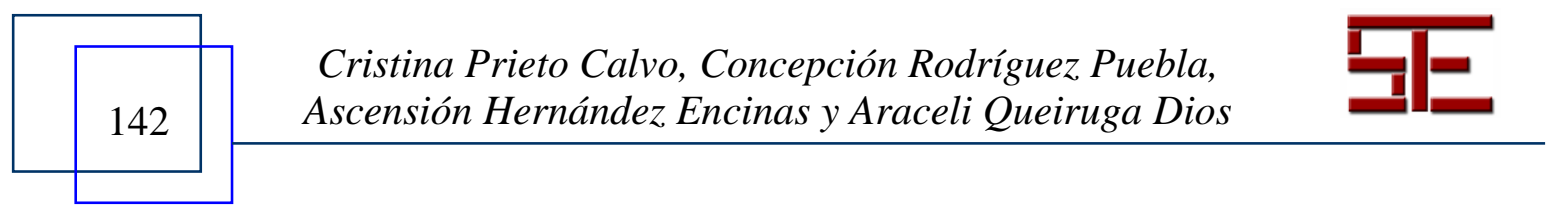




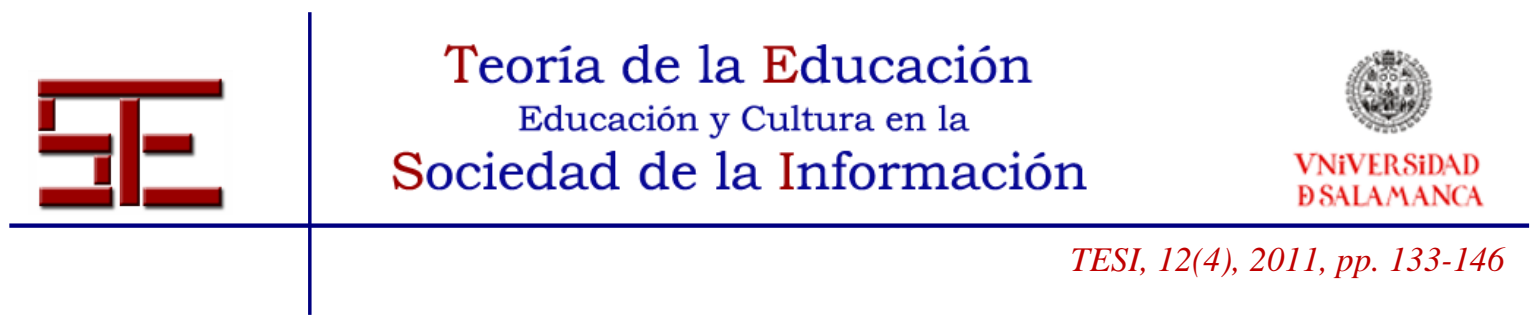

\section{2.- Climatología}

La colaboración con los estudiantes se desarrolla de la siguiente manera: para los estudiantes de Físicas se plantean preguntas sobre aspectos relacionados con los procesos climáticos y su caracterización mediante variables físicas. Los temas de los trabajos se centran en el análisis de los procesos físicos que describen y explican el clima y su variabilidad. Para los estudiantes de Ingeniería Agroalimentaria se plantean preguntas sobre riesgos climáticos en la agricultura y se proponen temas que abordan la variabilidad y el cambio climático desde la perspectiva agrícola para examinar las relaciones entre cultivos y clima. En las clases prácticas se preparan los datos climáticos y programas para realizar los estudios de variabilidad y de las simulaciones de procesos físicos. Todo ello se lleva a cabo con el soporte informático "GridAnalysisDisplaySystem" (GrADS) (Doty et al., 1995). Se seleccionan tareas que complementan los temas de las clases de teoría y prácticas. En la Figura 4 se presenta un momento de la exposición del trabajo.

a)

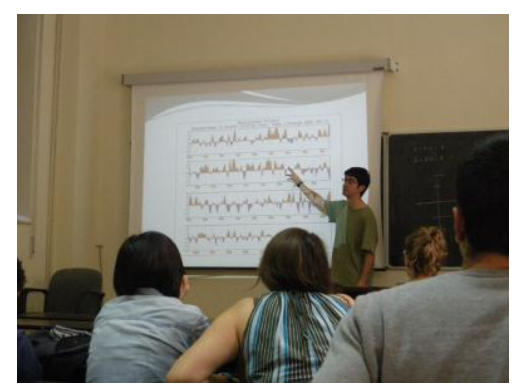

b)

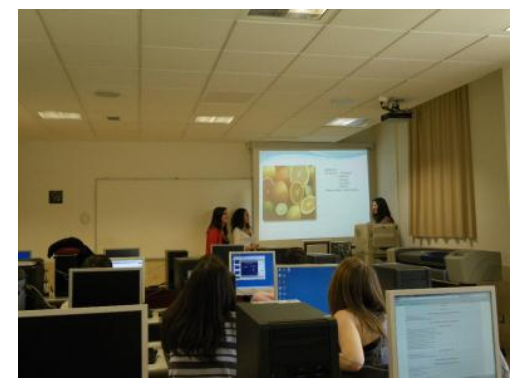

Figura 4: Estudiantes de: a) Física; b) Ingeniería Agroalimentaria, en la exposición del trabajo (mayo 2011).

La incorporación de la metodología colaborativa en el proceso de enseñanza respecto a los métodos de enseñanza tradicional requiere una gran dedicación por parte del profesor/a como son: la preparación del trabajo experimental, la corrección de las tareas, el diseño y orientación de los trabajos asignados a los grupos. Sin embargo, el trabajo es motivador por ser creativo, por las nuevas ideas que surgen al aplicar las diferentes herramientas TIC y reconocer lo que los estudiantes pueden hacer. La eficacia de esta metodología de enseñanza mediante asignación de trabajos en grupos se evidencia mediante las opiniones de los estudiantes. Destacando una mejor comprensión de los

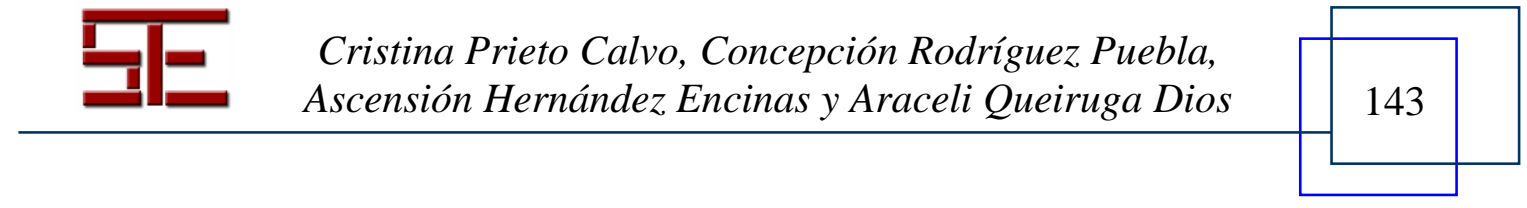




\begin{tabular}{c} 
Teoria de la Educación \\
Educación y Cultura en la \\
$\begin{array}{c}\text { VNiVERSIDAD } \\
\text { BSALAMANCA }\end{array}$ \\
\hline TESI, 12(4), 2011, pp. 133-146
\end{tabular}

fenómenos, en nuestro caso los climáticos, y la experiencia gratificante de exponer de forma oral trabajos porque supone un gran esfuerzo para aprender a estructurar las ideas $\mathrm{y}$ a presentarlas con claridad.

\section{3.- Ingeniería Industrial}

En la asignatura de Complementos de Matemáticas, que es optativa del $2^{\circ}$ ciclo de Ingeniería Industrial, se propone a los estudiantes la realización de un póster en el que muestren una aplicación a su especialidad de alguno de los métodos numéricos estudiados (Chapra-Canale, 2007). En la Figura 2 se muestra el ejemplo de un póster desarrollado sobre análisis modal.

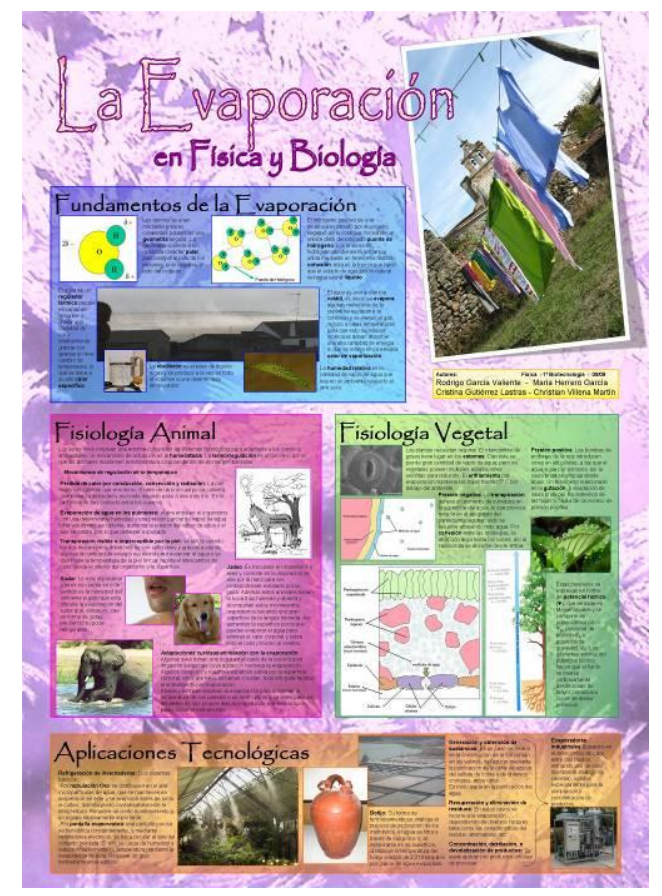

Figura 5: Póster desarrollado por estudiantes de $1^{\circ}$ de Biotecnología.

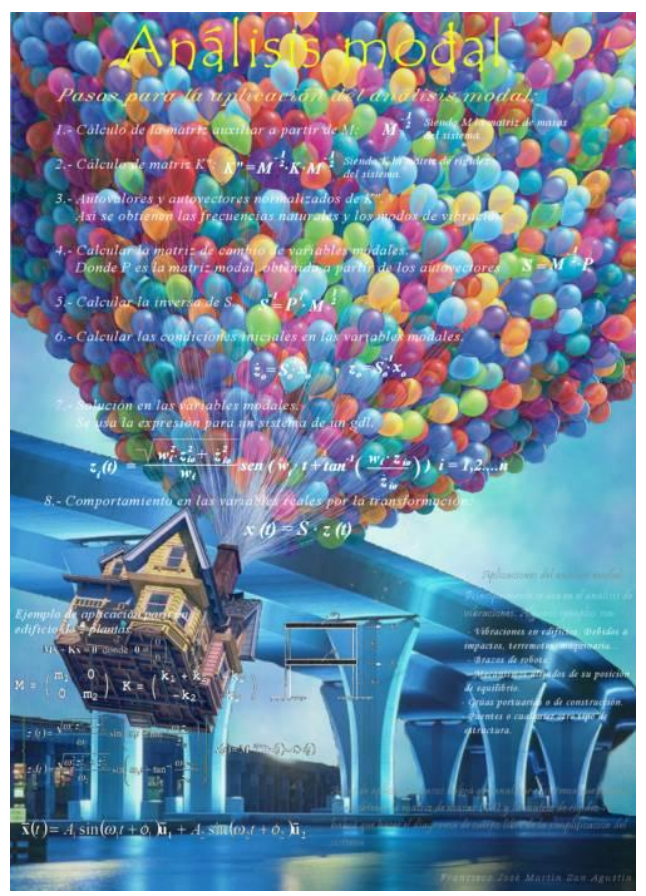

Figura 6: Póster desarrollado por estudiantes de $4^{\circ}$ de Ingeniería Industrial.

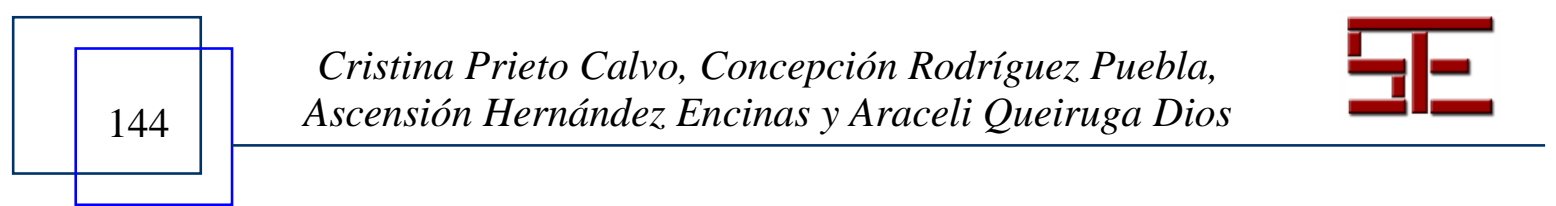




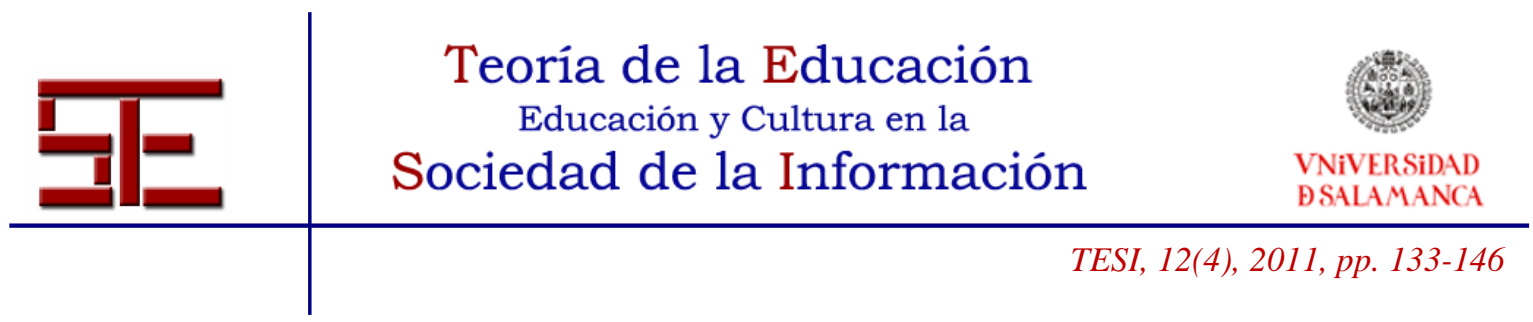

En esta asignatura en particular, se modelizan escenarios diversos de problemas de ingeniería con herramientas apropiadas para cada caso concreto, utilizando técnicas exactas o aproximadas. El curso se divide en clases de teoría, de problemas y prácticas informáticas con el paquete Mathematica (Wolfram, 1999), acabando con la exposición, por parte de los alumnos, de los pósters realizados.

\section{5.- CONCLUSIONES}

El trabajo de colaboración, apoyado por las TIC, completa la enseñanza tradicional, permitiendo que el estudiante desarrolle el autoaprendizaje, preparándole para su futura labor profesional en equipo de trabajo y capacitándole para su formación a lo largo de la vida.

Mediante estas experiencias colaborativas los alumnos se aproximan a la metodología del trabajo de investigación y reconocen la importancia del pensamiento crítico. La realización del trabajo científico les capacita para ser críticos con el trabajo de sus compañeros y a su vez positivamente competitivos. Con frecuencia manifiestan su deseo de profundizar y continuar el aprendizaje mediante búsqueda de información bibliográfica y a través de la web. El seguimiento y ayuda de los profesores les anima en su trabajo, considerándolo, junto con la calificación, un reconocimiento a su esfuerzo.

Introducir trabajos en grupo en la metodología docente da lugar a clases más dinámicas, favoreciendo las preguntas y discusiones, haciendo a los alumnos más participativos y fomentando su asistencia.

La valoración positiva de la experiencia por parte de profesores y alumnos nos anima a seguir considerando la combinación de actividades de trabajo en grupo y TIC como una metodología valiosa en el desarrollo de las distintas materias. Con objeto de enriquecer la experiencia, en el futuro inmediato incorporaremos otros recursos, como la pizarra digital, o herramientas de la web 2.0 que hagan más fluida la comunicación en el grupo. A más largo plazo probablemente se podrán aprovechar también las nuevas ventajas (todavía insospechadas) que sin duda aportará con su imparable desarrollo el mundo de las TIC.

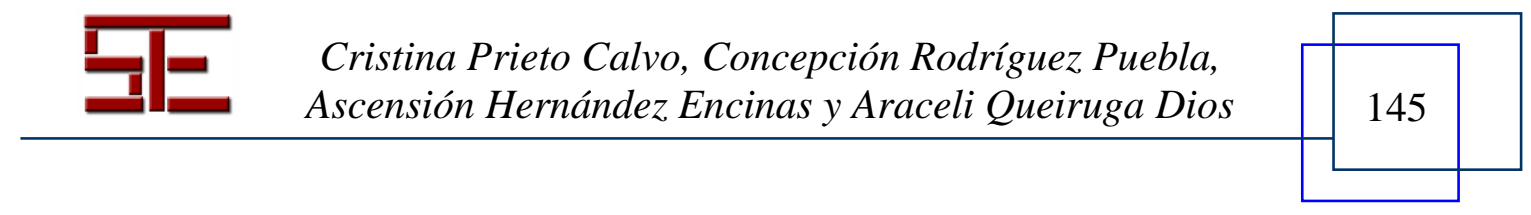




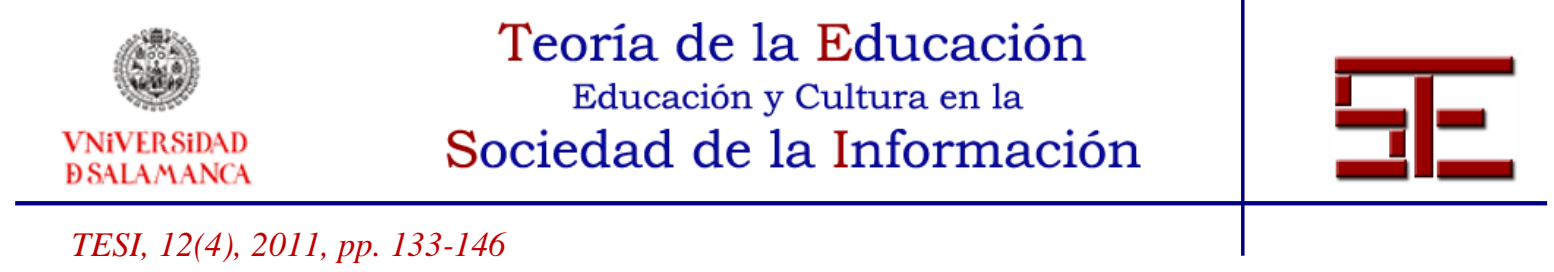

Resumiendo, mediante el trabajo colaborativo se incrementa la eficacia del aprendizaje porque aumenta el interés, mejora la autoestima, estimula el uso del lenguaje, promueve la coordinación y se aprende haciendo.

\section{BIBLIOGRAFÍA}

Chapra, S. C. \& Canale, R. P. (2007). Métodos Numéricos para Ingenieros. $5^{\mathrm{a}} \mathrm{ed.}$ McGraw Hill Interamericana.

Doty, B., Holt, T. \& Fiorino, M. (1995). The Grid Analysis and Display System $(G r A D S)$. Institute of Global Environment and Society. USA. Disponible en: http://www.iges.org/grads/.

Guitert, M., Romeu, T., Pérez-Mateo, M. (2007). Competencias TIC y trabajo en equipo en entornos virtuales. RUSC, 4 (1). Disponible en: http://rusc.uoc.edu.

Martínez M. \& Carrasco, S. (Coords.) (2006). Propuestas para el cambio docente de la universidad. Barcelona: Octaedro.

Queiruga Dios, A., Hernández Encinas, A., Visus Ruiz, I. \& Martín del Rey, A. (2011). A virtual Colaborative Environment Helps University Students to Learn Maths. Enterprise Information Systems - Lecture Notes in Business Information Processing, 600-606.

Wolfram, S. (2003). The Mathematica book (5 ${ }^{\text {th }}$ ed.). Wolfram Media. Cambridge University Press.

Para citar el presente artículo puede utilizar la siguiente referencia:

Prieto Calvo, C., Rodríguez Puebla, C., Hernández Encina, A. y Queiruga Dios, A. (2011). Experiencias docentes de trabajo colaborativo en distintas áreas de ciencias. Revista Teoría de la Educación: Educación y Cultura en la Sociedad de la Información. 12(4), 133-146 [Fecha de consulta: dd/mm/aaaa].

http://campus.usal.es/ revistas_trabajo/index.php/revistatesi/article/view/8530/8616

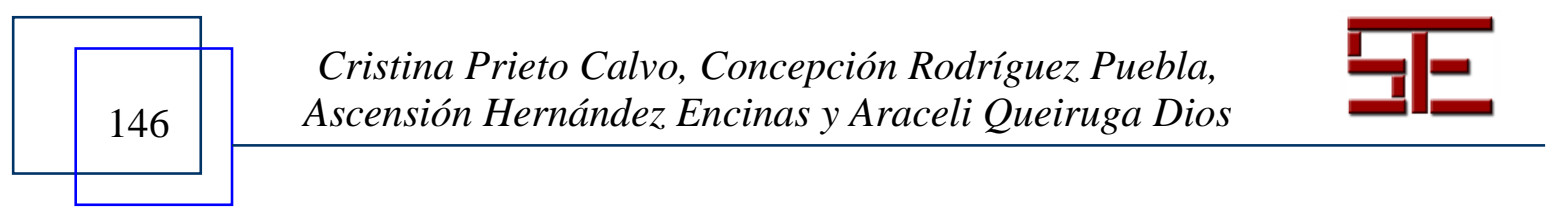

